\title{
EXAMPLES FOR THE INDUSTRIAL USE OF SYNCHROTRON RADIATION
}

\author{
J. Hormes, R. Chauvistré, W. Schmitt and M. Pantelouris \\ Physikalisches Institut der Universität Bonn \\ Nussallee 12, D-5300 Bonn 1, Germany
}

Synchrotron radiation has become a valuable tool for many fields of basic research. Several of the methods which were here developed are also suitable for solving industrial problems. Perhaps the most promising ones are the various X-ray techniques, e.g. X-ray absorption spectroscopy (EXAFS and XANES) and X-ray fluorescence. In the following article the theoretical and experimental basis for these techniques is shortly introduced and some instructive examples for such applications for actual industrial problems are dicussed. In the last section, X-ray lithography for the production of microstructures is presented as an example where synchrotron radiation is already today used for industrial production.

PACS numbers: 07.85.+n, 29.20.-c, 32.30.Rj, 78.70.Dm

\section{Introduction}

During the last twenty years synchrotron radiation has become a very important tool in basic research because of its unique properties [1]. Spectroscopic investigations with synchrotron radiation are carried out not only in physics, but also in chemistry, biology and material sciences. The results of these investigations are relevant, e.g. in the field of microelectronics, semiconductor technology, high-temperature superconductors, storage media, environmental techniques and several other fields belonging to what is called "high technology". Although several of the methods developed in basic research have already been optimized to the extent that application on a routine basis for industrial problems is possible without further improvements, the industrial use of synchrotron radiation in Europe and especially in Germany is still in its infancy. Outside of the Europe, the situation has already changed: several companies use synchrotron radiation at existing storage rings and in the USA and Japan several storage rings for mainly industrial use are planned or are already under construction. In most of these cases radiation will be used chiefly for X-ray applications in the field of microelectronics.

Instead of discussing all possible industrial applications of synchrotron radiation it is the aim of this contribution to give only some instructive examples. All of 
these experiments were carried out at the electron accelerators of the Physikalisches Institut der Universität Bonn in collaboration with industrial companies. These examples are believed to be quite typical for further applications, since in all cases the advantages offered by synchrotron radiation over other light sources are used consistently. Due to the specific selection of examples only a limited section of all possible applications will be considered. Thus, it seems important to mention that there are several other techniques of major industrial importance not discussed here, e.g. diffraction and scattering techniques (small-angle and wide-angle scattering) as well as the different techniques of electron spectroscopy (UPS, XPS etc.) which are routinely used with conventional light sources in industrial laboratories.

\section{Application of X-ray absorption spectroscopy: EXAFS and XANES measurements}

\subsection{Basic physics and experimental setup}

\subsubsection{Basic physics}

$\mathrm{X}$-ray absorption spectroscopy (XAS) with synchrotron radiation over the last years has become a valuable tool for the analysis of the electronic and geometric structure of non-crystalline materials on an atomic scale. In these measurements the photoabsorption cross-section near an X-ray absorption edge of an atom (in most cases the $K$ - or the $L_{11}$-edge) is determined as a function of the energy of the exciting radiation. The spectra obtained in this way show that the atomic absorption is modified by solid state effects that lead to a fine structure below and up to several hundred $\mathrm{eV}$ above the corresponding ionisation edge. As a typical example the sulphur $K$-shell absorption spectrum of $\mathrm{S}_{2} \mathrm{Cl}_{2}$ is shown in Fig. 1. As marked

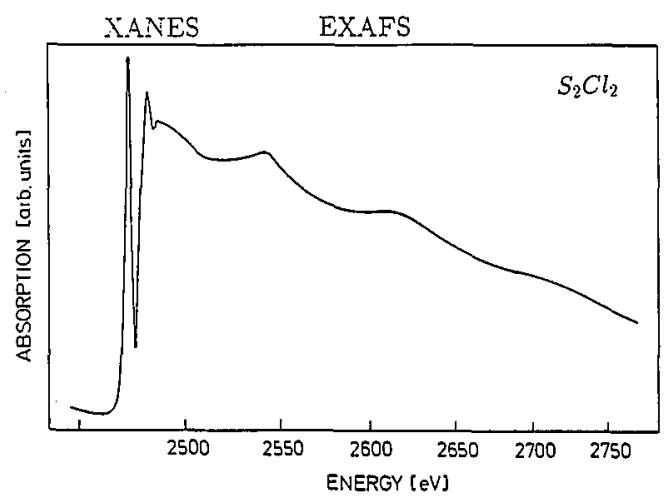

Fig. 1. The sulphur $1 s \mathrm{X}$-ray absorption spectrum of $\mathrm{S}_{2} \mathrm{Cl}_{2}$.

in this figure, two regions can be distinguished in the spectra: the structure close to the absorption edge (XANES = X-ray absorption near-edge structure) and the high-energy structure (EXAFS = extended X-ray absorption fine structure). 
The EXAFS is caused by an interference of the spherical wave describing the outgoing photoelectron with parts of this wave that were backscattered from neighbouring atoms. The resulting interference pattern can be described by a closed analytical form. The analysis of the phase of the interference pattern provides very accurate information on radial distances between the absorbing atom and the first coordination shells neighbour atoms. From the amplitude of the interference structure information on the type and the number of the surrounding atoms can also be derived, though with much less accuracy than the information about the radial distances. Details of the various techniques for measuring EXAFS and the process of data analysis are discussed extensively in the literature [2-4].

XANES incorporates the structure below the ionisation potential (IP) as well as the structure extending about some tenths eV above the IP. Spectral features below the IP correspond to transition of the excited electron to unoccupied molecular orbitals; the region above the IP is dominated by multiple scattering effects of the outgoing electron wave. This part of the spectrum contains information on the geometrical arrangement of the atoms around the absorbing atom and on the electronic structure of this atom [5]. As this part of the spectrum cannot be described analytically, the available information has to be extracted by a "fingerprint" method, comparing the spectra of well-characterized reference compounds with the spectrum of the sample under investigation. For the interpretation of XANES spectra some useful empirical rules exist which have been derived by systematic investigations of well-known model compounds. Perhaps the most important is the observation that the so-called "edge" in an absorption spectrum (defined as the turning point in the rise of the absorption to the continuum) is shifted towards higher energies as a function of increasing valency of the absorbing atom (and increasing electronegativity of the neighbouring atoms) [6]. Another rule whose general validity is still being disputed, connects the energy position of the so-called shape resonances (the first and often rather strong absorption maxima above the IP, not yet belonging to the EXAFS region) with the radial distances of the absorbing atom to its next neighbours [7].

As compared to other techniques for structural analysis X-ray absorption spectroscopy has some very specific advantages:

- no long-range order is required;

- the local surroundings of each type of atom can be investigated separately;

- the investigations do not destroy the sample;

- in principle, locally resolved experiments are also possible;

- due to the penetration strength of X-rays, XAS experiments do not require a good vacuum which is absolutely necessary for experiments where electrons are detected.

Using suitable sample chambers with windows, e.g. made from kapton or beryllium the last property even allows in situ investigations of samples in e.g. a liquid or a special gas atmosphere. This is of major importance, e.g. for investigating liquids, for high-pressure measurements and for the in.situ observation of catalytic reactions. 
In the following two examples for XAS (that only consider the XANES region) are given, showing how typical industrial problems can be solved using the advantages of synchrotron radiation.

\subsubsection{Experimental setup}

The X-ray absorption experiments discussed in the following sections were performed at the electron storage ring ELSA of the Physikalisches Institut der Universität Bonn. The experimental setup used for these measurements is shown schematically in Fig. 2. This arrangement can be regarded as typical and is used

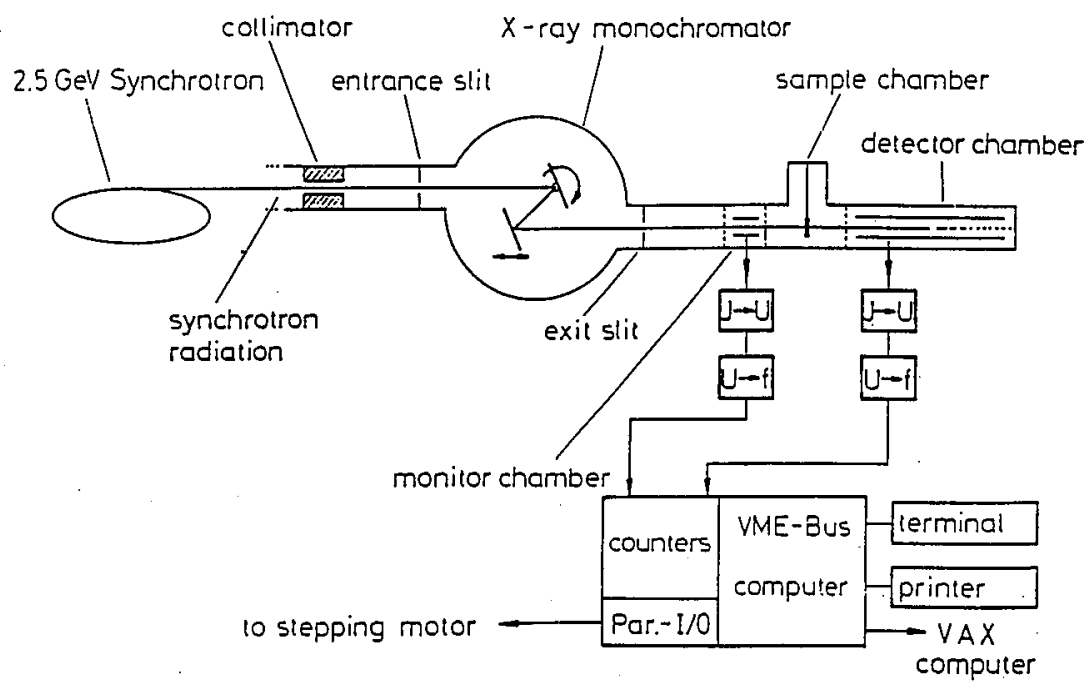

Fig. 2. Schematic drawing of an experimental setup for measuring X-ray absorption spectra.

with slight modifications in all synchrotron radiation laboratories. The "white" light emitted from the storage ring enters a double crystal monochromator (here of the Lemmonier type [8]) employing two perfect single crystals. The photon energy is varied by changing the Bragg angle in the monochromator. This particular type of monochromator provides a monochromatized beam of fixed direction and position. The primary and respective intensities falling on and transmitted by the sample are measured by means of ionisation chambers. The preamplified currents from the ionisation chambers are digitized and stored by a microcomputer system. By dividing the two signals and taking the logarithm of the result, a value directly proportional to the desired absorption coefficient is obtained. Depending on the crystals used, the energy range between about $900 \mathrm{eV}$ and $20 \mathrm{keV}$ can be covered. Thus, all elements with $Z>9$ can be investigated either at the $K$ or at the $L$ shell. The sulphur $K$-shell spectra shown in Figs. 3, 6 and 7, for example, were measured with InSb(111) crystals. Here the monochromatic flux is about $10^{9}$ photons/s and the transmission width about $1.0 \mathrm{eV}$. 


\subsection{Determination of the valency of chromium in chemical compounds}

Chemical compounds containing chromium are widely used in industrial applications, e.g. in several colour pigments, in tannins and for many corrosives. Whereas $\mathrm{Cr}(\mathrm{III})$ is considered not harmful, $\mathrm{Cr}(\mathrm{VI})$ is believed to be a strong carcinogen. Thus, it is important to rule out that there is any $\mathrm{Cr}(\mathrm{VI})$ in products for general use. It is of course possible to determine the valency of chromium in a compound using standard ESCA techniques. However, application of this technique meets severe experimental problems with a lot of actual products (e.g. leather in an UHV apparatus!) and also the results obtainable are not in all cases as clear cut as required, keeping in mind that chromium can also have valencies IV and $V$ [9]. The problem discussed here (determination of the valency of an element in a particular chemical compound) is very widespread in industry, and can be regarded as a standard problem of analytical chemistry. As was pointed out in Sec. 2.1.1 such a problem can be solved quite easily by looking at the near-edge spectra (XANES) of the element in question.

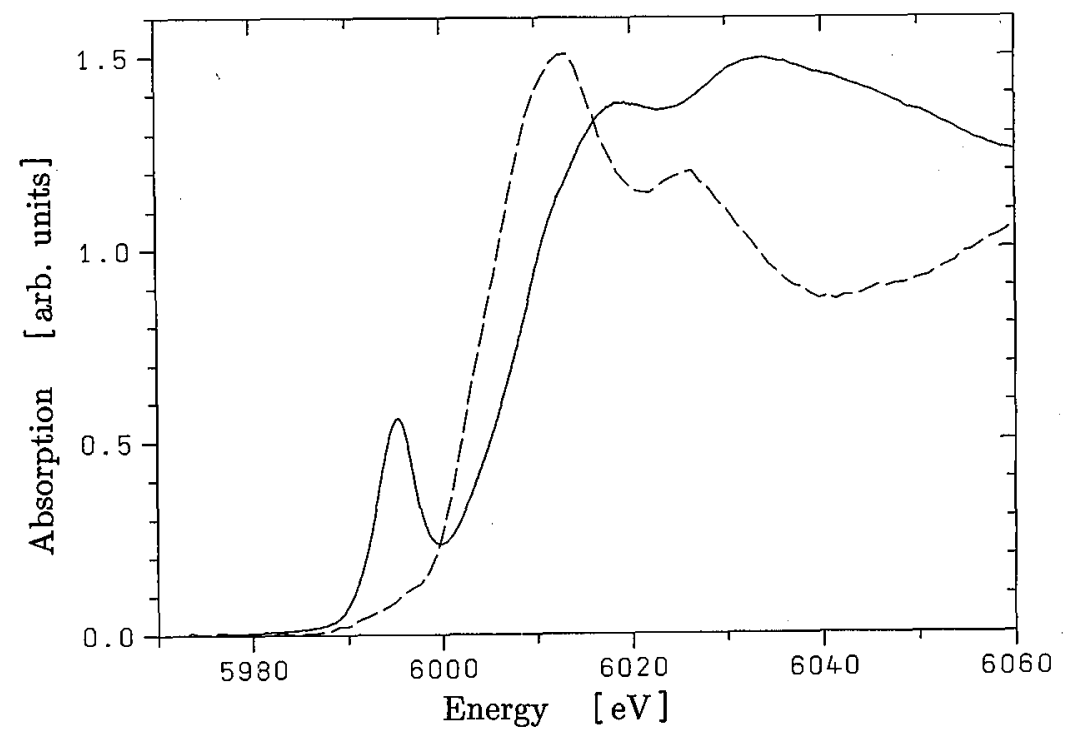

Fig. 3. X-ray absorption near-edge (XANES) spectra at the $\mathrm{Cr} K$-edge of $\mathrm{Cr}(\mathrm{VI}) \mathrm{O}_{3}$ $(---)$ and $\mathrm{Cr}(\mathrm{III})_{2} \mathrm{O}_{3}(-)$.

In Fig. 3, XANES spectra are shown of two suitable model compounds with well-known valency (in this case $\mathrm{Cr}(\mathrm{VI}) \mathrm{O}_{3}$ and $\mathrm{Cr}(\mathrm{III})_{2} \mathrm{O}_{3}$ ). Comparing these spectra two significant differences become evident:

- the spectrum of the compound with valency VI has a-distinct white line whereas the spectrum of the compound with valency III is flat in the pre-edge region; 


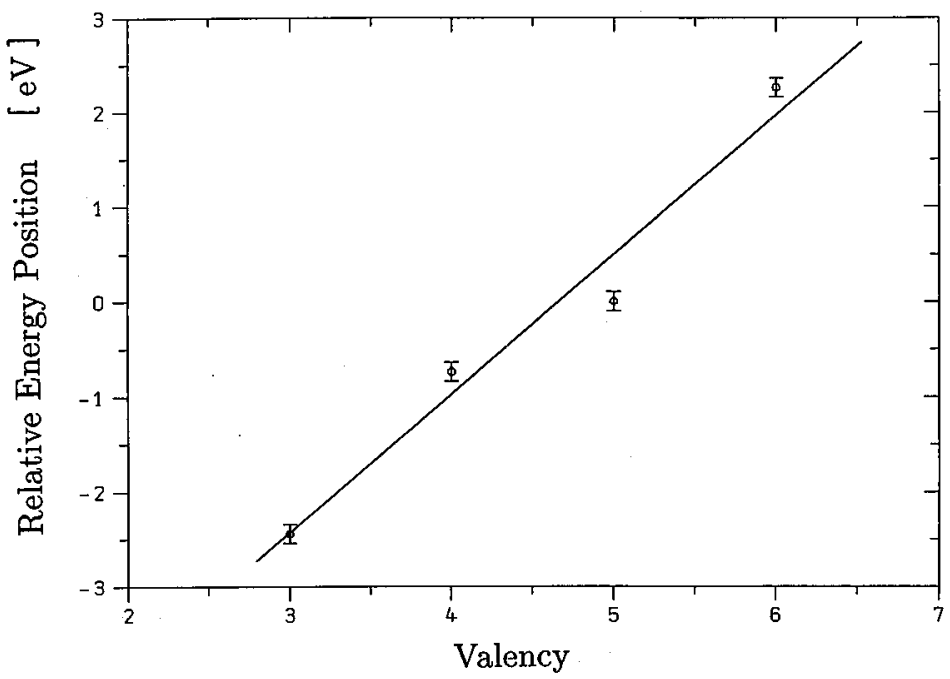

Fig. 4. Relative energy position of the "edge" in various chemical compounds containing chromium as a function of valency.

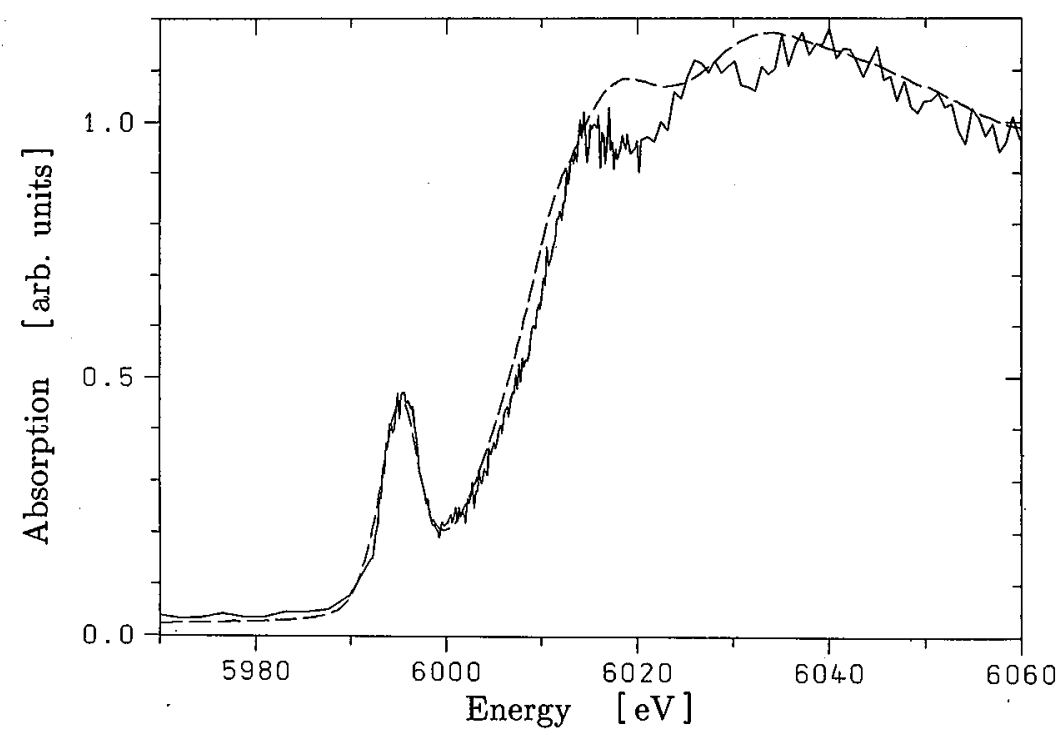

Fig. 5. X-ray absorption near-edge (XANES) spectra at the $\mathrm{Cr} K$-edge of $\mathrm{Cr}(\mathrm{VI}) \mathrm{O}_{3}$ $(---)$ and a colour pigment containing chromium (-).

- the "edge". is clearly shifted to higher energies in the chromium (VI) compound.

Further measurements of suitable chromium compounds with well-defined 
valencies IV and $\mathrm{V}$ confirm systematic trends in intensity and energy position of the white line and the edge, respectively. Plotting the energy position of the "edge" versus valency an approximate linear dependence is observed with an energy shift of more than $1 \mathrm{eV}$ per valency unit. This plot is shown in Fig. 4. A similar dependence is found when the energy position of the white line is plotted versus valency. With these results a determination of the valency of an unknown compound is possible without difficulty. A striking example is shown in Fig. 5. Here the Cr-XANES spectrum of a chromium colour pigment is compared directly with the spectrum of $\mathrm{Cr}(\mathrm{VI}) \mathrm{O}_{3}$. The energy position of the white line as well as the position of the edge are almost the same in both compounds. Thus, it is safe to say that the pigment investigated here contains at least a great part of chromium with valency VI.

Concluding this section it is worth mentioning that with very careful measurements a quantitative analysis of the ratio $\mathrm{Cr}$ (III)/Cr(VI) is also feasible and that an admixture of less than 1 percent of $\mathrm{Cr}(\mathrm{VI})$ in $\mathrm{Cr}(\mathrm{III})$ can still be identified.

\subsection{Time-dependent observation of sulphur vulcanisation of rubber}

Though sulphur vulcanisation of rubber was invented more than 150 years ago by Goodyear, the chemical processes taking place during vulcanisation are still not completely clarified. This is especially true for the processes involved in the creation and the disintegration of the sulphur chains forming the cross links between the polymer chains, and thus being chiefly responsible for the mechanical properties of the rubber product. Besides other problems of rubber chemistry [10-12], this aspect is investigated by taking XANES spectra at the sulphur $K$-edge. As recording such a spectrum takes less than 3 minutes, it is possible to follow the vulcanisation process in real time by taking a spectrum every few minutes.

To answer the above question, it is crucial that the XANES spectra at the $\mathrm{S} K$-edge allow the discrimination of sulphur chains of different length. This is demonstrated in Fig. 6 where $S K$-edge XANES spectra of suitable model compounds $\mathrm{R}-\mathrm{S}_{\boldsymbol{x}}-\mathrm{R}(\boldsymbol{x}=1,2,3)$ (in this example $\mathrm{R}=$ methyl) are shown. For the monosulfidic chain there is just one white line which can be assigned to a transition into a $\sigma^{*}(\mathrm{C}-\mathrm{S})$ orbital. For the disulfidic chain, a second more intense resonance appears at the low-energy side which is now assigned to a transition into a $\sigma^{*}(\mathrm{~S}-\mathrm{S})$ orbital [13]. Due to further changes in the surroundings the intensity of these resonances of the S-S transition is shifted to lower energies as a function of increasing chain length as can be seen from the XANES of the trisulfidic bridge.

In Fig. 7 some $S K$-XANES spectra are shown which where taken during the vulcanisation process as a function of time (and temperature). The temporally first spectrum (the one at the bottom of Fig. 6) was taken before the sample was actually heated. From the intensity and energy position of the white line in this spectrum it can be concluded that some chemical reactions leading to polysulfidic chains have already taken place in the mixture of rubber, sulphur in the form of $\mathrm{S}_{8}, \mathrm{ZnS}$ and an accelerator. The heating of the sample prepared as a thinly cut film causes a further increase in longer sulphur chains which can be inferred from the increasing intensity of the $\sigma^{*}(\mathrm{~S}-\mathrm{S})$ resonance. During the next steps of the 

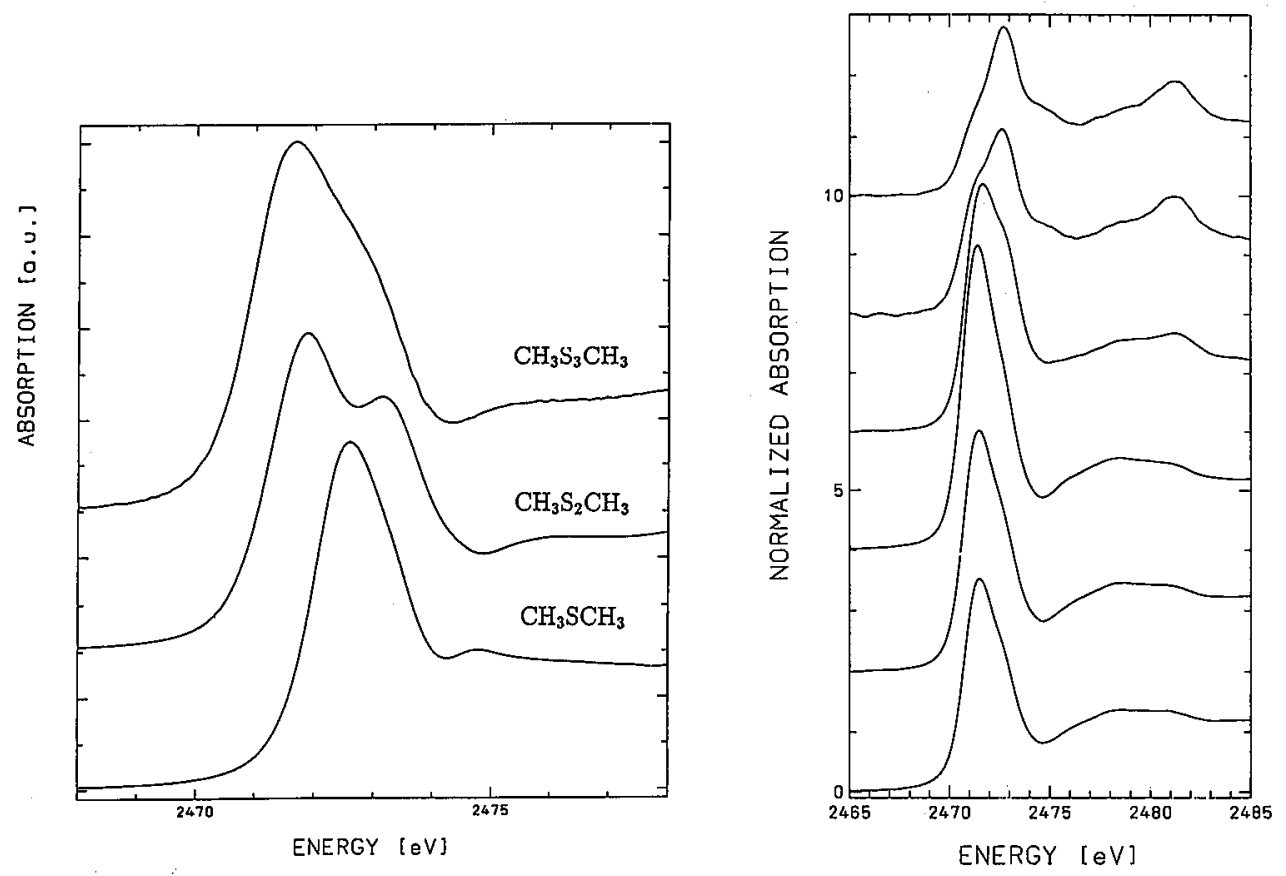

Fig. 6. X-ray absorption near-edge (XANES) spectra at the sulphur $K$-edge of model compounds with the structure $\mathrm{R}-\mathrm{S}_{\boldsymbol{x}}-\mathrm{R}(x=1,2,3)$.

Fig. 7. Sulphur $K$-XANES spectra obtained time dependent during the vulcanisation of rubber; time sequence starting from the bottom to the top.

reaction, already a slight shift of the absorption maximum to higher energies can be observed reflecting a first disintegration of chains. Spectral changes in the energy region of about $2473 \mathrm{eV}$ are caused by the production of species containing some parts of the accelerator molecules. These processes continue during the following spectra until in the last spectrum the position of the white line clearly indicates a large admixture of monosulfidic sulphur. In addition to the spectral changes at about $2472 \mathrm{eV}$, further changes can be observed in the energy region of about $2481.5 \mathrm{eV}$. Again by comparison with the spectra of suitable model compounds this structure can be assigned to transitions in zinc sulfate. These features thus are a first indication of the beginning of the ageing of the rubber.

Measurements similar to the one here discussed can be applied not only for the investigation of chemical reactions, but also to investigate the various influences of accelerator molecules, ageing protectives and other additives.

\section{Angle-resolved, self-ratio X-ray fluorescence spectroscopy}

The determination of the depth profile of concentration of a component in the surface region of a material is a major concern of surface and thin-film analysis [14]. A new apparatus for depth-profile and thin-film analysis using angle-resolved, 
self-ratio X-ray fluorescence spectroscopy (AR/SR/XFS) with synchrotron radiation is in its final stage of development at the beamline BN2 of the ELSA storage ring. The objects of interest are primarily ion-implanted semiconductors for use as reference materials. Each reference material is to be investigated for the centroid depth of the ion-implanted dopant as well as for the implantation dose density. These investigations are being undertaken within a research contract with the Research Institute of the Deutsche Bundespost Telekom as part of the multinational Versailles project on Advanced Materials and Standards (VAMAS) [15, 16]. In the beginning AR/SR/XFS had been developed for use with conventional X-ray spectrometers using X-ray tubes. A decisive advantage of AR/SR/XFS over ion-beam methods such as secondary-ion mass spectroscopy (SIMS) or Rutherford backscattering spectroscopy (RBS), in detail discussed elsewhere [19], is that AR/SR/XFS is non-damaging and can therefore be used for calibration purposes on the same reference material repeatedly.

Synchrotron radiation, because of its brightness and intensity is used to overcome the disadvantage of a low signal-to-background ratio for determination of implantation dose densities less than several $10^{15} \mathrm{~cm}^{-2}$. With synchrotron radiation measurements on samples with implantation dose densities as low as $10^{14} \mathrm{~cm}^{-2}$ are possible [20]. The principle of AR/SR/XFS, which has been discussed in detail by Gries et al. [17, 18, 22], is based upon the angle-dependent attenuation of the excited fluorescence radiation in the sample. The fluorescence intensity of the element of interest is measured at different take-off angles. If the type of depth profile or the ratios of its distribution moments are known, it is possible to determine the centroid depth of the profile by measurement of the dopant signal at two different angles of take-off. The method allows investigations of implanted and diffused depth profiles as well as of thin films in the sub-micron range [18, 22]. The apparatus is shown schematically in Fig. 8. It is based upon a commercial, wavelength-dispersive sequential X-ray spectrometer (SIEMENS SRS1). The incoming, "white" synchrotron radiation passes a monitor ionisation chamber and irradiates the sample mounted on a goniometric cradle. The sample can be tilted within a range of $90^{\circ}$ in steps of $0.01^{\circ}$. The angle between the incoming radiation and the direction of the detector, defined by a slit of $1 \mathrm{~mm}$ width and $150 \mathrm{~mm}$ length, is about $55^{\circ}$. The energy resolution of the spectrometer is defined by the width and length of the Soller slit and the rocking curve of the Bragg crystal used. Using a graphite crystal $(2 d=6.708 \AA)$ we found an energy resolution of about $12 \mathrm{eV}$ for phosphorus $K_{\alpha}$ radiation. In contrast to energy-dispersive detection, e.g. with a $\mathrm{Si}(\mathrm{Li})$-detector, wavelength-dispersive detection as was used here allows full separation of fluorescence lines even from direct neighbours in the periodic table such as silicon, phosphorus or sulphur. It was demonstrated that it is not possible to trace low doses of phosphorus in a silicon substrate with a $\mathrm{Si}(\mathrm{Li})$-detector [20].

The fluorescence radiation is detected by a gas flow proportional counter and the counting events are stored by a VME-bus computer which also controls the stepping motors for the sample tilt and the $2 \vartheta$ rotation of the analyzing crystal. The HeNe-laser is used for the calibration of the angle of incidence [19, 20]. Figure 9 shows the calculated fluorescence intensity ratio corresponding to take-off angles of $15^{\circ}$ and $75^{\circ}$ versus the centroid depth for $\mathrm{P} K_{\alpha}$-radiation in a silicon substrate. 


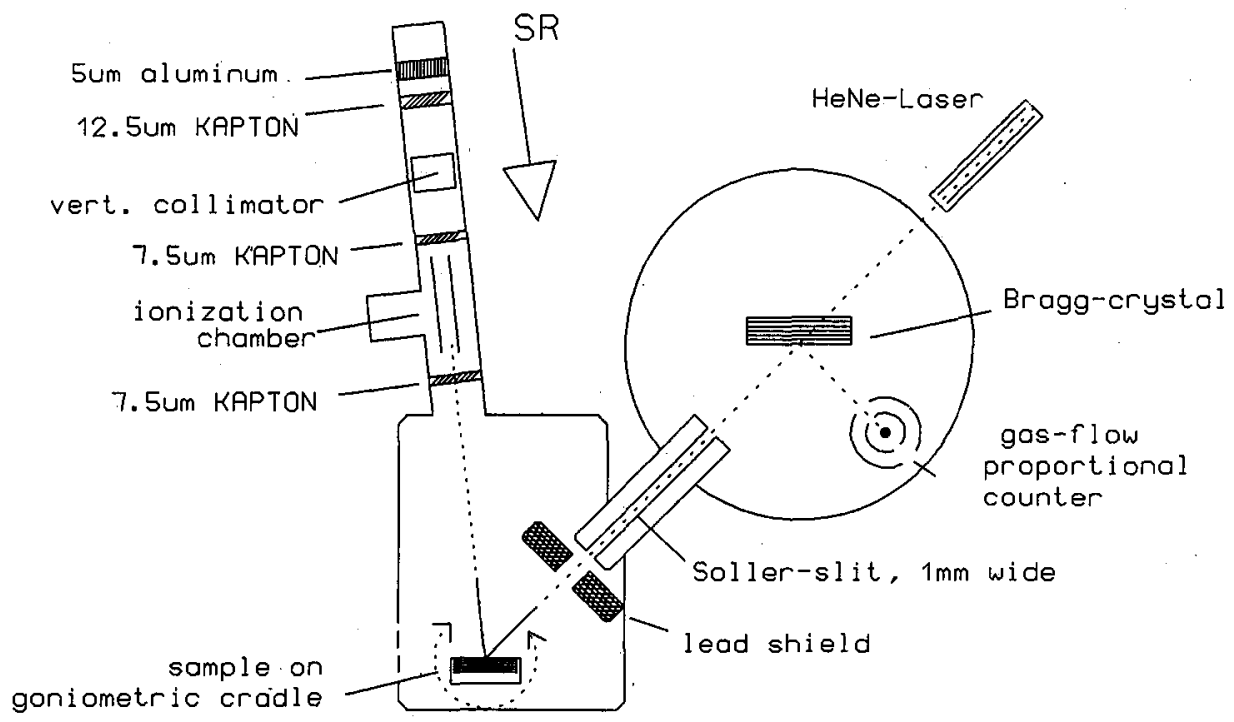

Fig. 8. Schematic drawing of the experimental setup for measuring AR/SR/XFS.

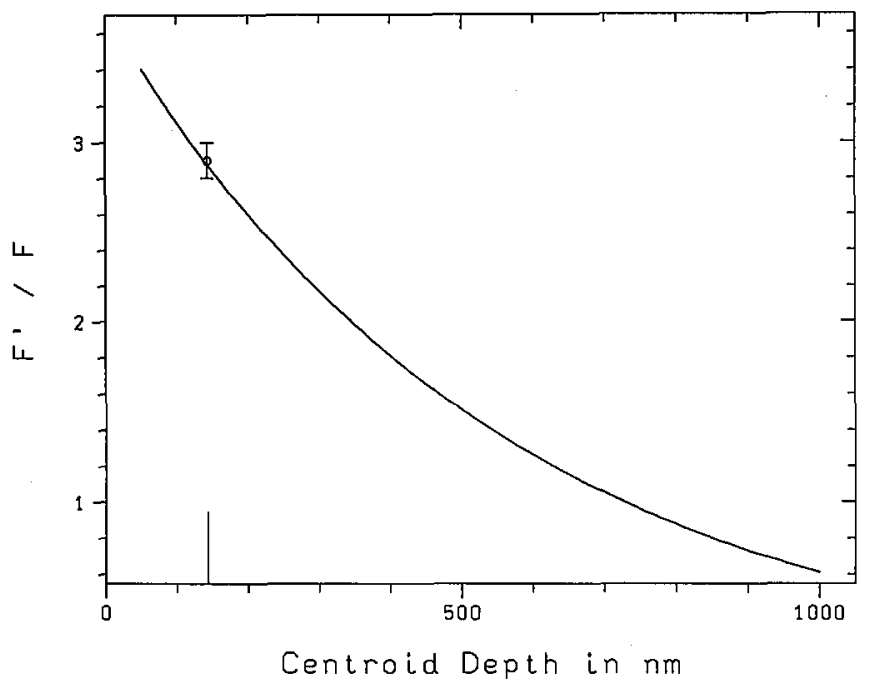

Fig. 9. Fluorescence ratios $F^{\prime} / F$ for $\mathrm{P} K_{\alpha}$-radiation in silicon corresponding to take-off angles of $15^{\circ}$ and $75^{\circ}$ versus the centroid depth calculated for a Gaussian distribution of the depth profile.

The implantation energy was $100 \mathrm{keV}$ and the implanted dose of phosphorus was $10^{16} \mathrm{~cm}^{-2}$. A Gaussian distribution of the depth profile was assumed for the calculation of the ratios and the evaluation of the experimental values. The value at 
the ratio of $2.90 \pm 0.09$, resulting from some preliminary measurements, corresponds to a centroid depth of $(144 \pm 18) \mathrm{nm}$. In spite of the preliminary state of these measurements, the result - within its error margins - is in accordance with measurements on the same sample using AR/SR/EMA and AR/SR/XFS with $\mathrm{X}$-ray tubes $[17,21,22]$.

Here only the application of AR/SR/XFS for the investigation of depth profiles of ion-implanted semiconductors was discussed. However, this method has much more technical applications, e.g. the investigation of buried thin films and of the diffusion profiles of surface protective layers and metallic electrical connections on semiconductors, just to mention a few of those.

\section{X-ray lithography for the production of microstructures}

In this application synchrotron radiation is not used for analytical purposes but for the production of microstructures within the so-called LIGA process (in German: Lithographie, Galvanoformung, Abformtechnik). The LIGA process was developed in the early eighties in the Institut für Kernverfahrenstechnik of the Nuclear Research Center in Karlsruhe (KfK) for the production of micron-sized nozzles for uranium isotope separation [23].

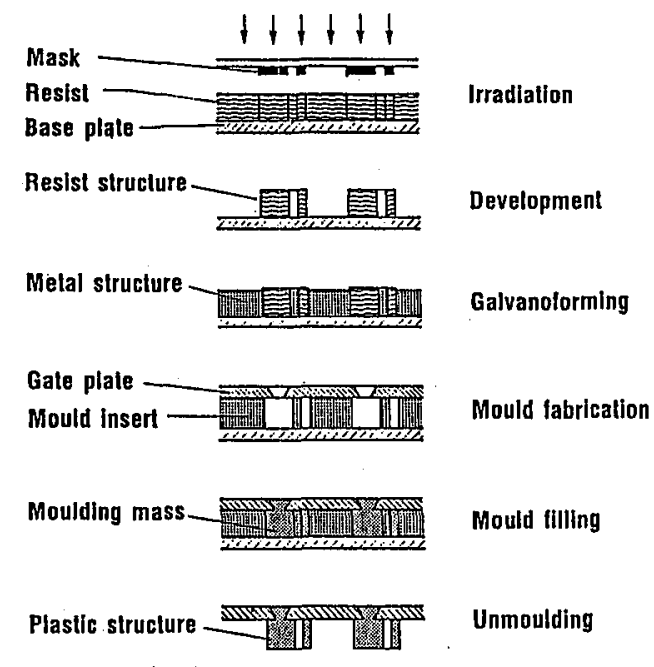

Fig. 10. Schematic representation of the process sequence of the LIGA method.

Figure 10 shows schematically the process sequence of the LIGA method. The first process step in which synchrotron radiation is used is deep-etch X-ray lithography. Here a special X-ray resist with a height of up to several hundred micrometers is exposed through an X-ray mask to very intense parallel $\mathrm{X}$-rays. The X-ray resist is usually a polymeric material (until now PMMA = polymethylmethacrylate) whose dissolution rate changes in a liquid solvent after high-energy irradiation. Thus, those parts of the resists which are not protected by the absorbing pattern on the mask are dissolved after irradiation whereas the protected parts 
remain undamaged. With this process quasi-three-dimensional microstructures are produced in PMMA. These structures can either be the final product or it can be further processed.

In the second step the PMMA structure is used as a template in an electroforming process where metal is deposited onto the electrically conductive substrate in the spaces between the resist structures. In this way, a metal structure being complementary to the PMMA structure is obtained. This metal structure can now be the final product or again be used in the third process step as a mold insert for multiple reproduction of the original structure by means of a molding process.

Lateral dimensions of the structures produced are in the micrometer region with structural heights of up to several hundreds of micrometers. Thus, with the LIGA method aspect ratios (= ratio of height and smallest lateral dimension) up to 100 can be obtained whereas in thin film lithography as used in microelectronics aspect ratios of 2 to 3 are typical. Three properties of synchrotron radiation are essential for performance of deep-etch X-ray lithography:

- the high degree of collimation,

- the high energy density and

- the high spectral density at short wavelengths.

The small divergence is in view of some degrading mechanisms (Fresnel diffraction, high-energy photoelectrons produced by the X-rays and the time evolution of the profiles during the developing process) essential for the precision of the structures produced by the lithographic process. This precision is illustrated in Fig. 11 which shows a scanning electron micrograph of a test structure where the width of a bar has been determined by means of a critical dimension measuring system. Over a height of about 330 micrometers the width varies less than $\pm 0.1 \mu \mathrm{m}$ [24].

The high energy density of synchrotron radiation provides the radiation dose necessary for the desired chemical modifications in the irradiated parts of the resist within a "reasonable" time. As the resist PMMA used until now is not very sensitive, an irradiation of a structure of 300 micrometers thickness and a width of $10 \mathrm{~mm}$ still takes about half an hour at the storage ring ELSA in Bonn running with (at the moment) typical parameters $(2.3 \mathrm{GeV}$, a verage current $50 \mathrm{~mA}$ ).

The wavelength of the radiation used for the irradiation has to be adapted carefully to the height of the corresponding sample so that the radiation induced processes necessary for the development process can take place to a sufficient degree over the total height. If the wavelength of the radiation is too long, most of the energy is already absorbed in the upper layers of the resist; if the wavelength is too short, there is only low absorption over the whole depth of the resist. For heights of several hundred micrometer produced till now, a characteristic wavelength $\lambda_{c}$ for the synchrotron radiation of about $0.2 \mathrm{~nm}$ is required. This in turn can only be obtained by circular accelerators running at an electron energy of about $2.5 \mathrm{GeV}$.

Based on the LIGA method a large number of innovative microstructures for various fields of application can be produced. Some of these fields are: micromechanics, process technology, energy technology, medicine and biotechnology, sensors, microoptics and microelectronics. For all these fields concepts of some products have been worked out and most of these were already manufactured on 

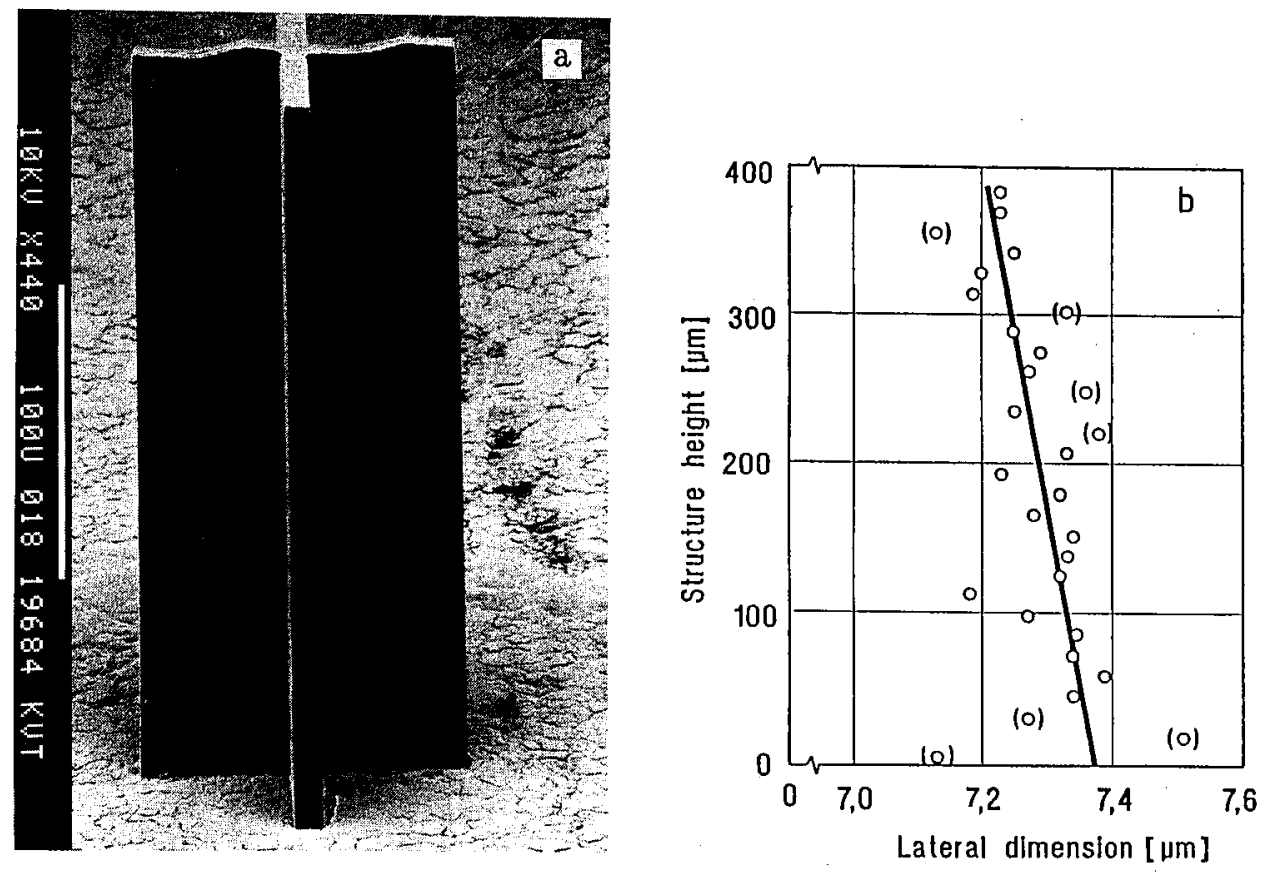

Fig. 11. a) Scanning electron micrograph of a crossed-shaped test structure. b) Bar width of the test structure determined by a critical dimension measuring system.

a laboratory scale. Quite recently, movable microstructures have also been produced thus making it possible to manufacture sensors and actuators. An impressive example of these possibilities is a microturbine which achieved up to 2000 revolutions per second and a lifetime of more than two hours [25]. At present, the LIGA method is being further refined at the Institut für Mikrostrukturtechnik of KfK, Karlsruhe, and by the IMM, Institut für Mikrotechnik, Mainz, whereas the commercial realisation has been taken over by a specially founded company named MicroParts.

\section{Acknowledgements}

Some of the experimental developments leading to the above described industrial applications have been supported by the Bundesminister für Forschung und Technologie. The support of our industrial partners and of the Research Institute of the Deutsche Bundespost Telekom is gratefully acknowledged. The authors express thanks to the Institut für Mikrostrukturtechnik of KfK, Karlsruhe, for permission to use their figures. 


\section{References}

[1] The properties of synchrotron radiation and several of the techniques for which synchrotron radiation is used are discussed in the corresponding handbooks, e.g.: Handbook of Synchrotron Radiation, Vol. 1-4, Eds. D.E. Eastman, Y. Farge, North-Holland, Amsterdam 1983-1991; Application of Synchrotron Radiation, Eds. C.R.A. Catlow, G.N. Greaves, Blackie, Glasgow 1990.

[2] E.A. Stern, Contemp. Phys. 19, 289 (1978).

[3] B.K. Teo, D.C. Joy, EXAFS Spectroscopy, Plenum Press, New York 1981.

[4] X-Ray Absorption, Principles, Applications, Techniques of EXAFS, SEXAFS and $X A N E S$, Ed. D.C. Koningsberger, R. Prins, Wiley, New York 1988.

[5] A. Bianconi, in Ref. [4], p. 573.

[6] B. Lengeler, Phys. Bl. 46, 50 (1990).

[7] F. Sette, J. Stöhr, A.P. Hitchcock, J. Chem. Phys. 81, 4906 (1984).

[8] M. Lemonnier, O. Collet, C. Depautex, J.M. Esteva, D. Raoult, Nucl. Instrum. Methods 152, 109 (1978).

[9] H. Plum, GIT 9, 969 (1991).

[10] R. Chauvistré, U. Kuetgens, J. Hormes, G. Weymans, Sacietà Italiana Di Fisica, Conf. Proc., 2nd European Conf. Prog. X-Ray Synchrotron Radiat. Res., Vol. 25, 879 (1990).

[11] R. Chauvistré, Ph.D. thesis, Bonn University, 1992.

[12] R. Chauvistré, J. Hormes, D. Brück, K. Sommer, H.-W. Engels, Kautsch. Gummi Kunstst., in press, 1992.

[13] A.P. Hitchcock, S. Bodeur, M. Tronc, Physica B 158, 257 (1989).

[14] Thin Film and Depth Profile Analysis, Topics in Current Physics, Vol. 37, Ed. H. Oechsner, Springer, Berlin 1984.

[15] C.J. Powell, Surf. Interface Anal. 11, 103 (1988).

[16] W.H. Gries, J. Vac. Sci. Technol. A 7, 1639 (1989).

[17] W.H. Gries, F.T. Wybenga, Surf. Interface Anal. 3, 251 (1981).

[18] W.H. Gries, Fresenius Z. Anal. Chem. 329, 133 (1987).

[19] W. Schmitt, J. Hormes, U. Kuetgens, W.H. Gries, Rev. Sci. Instrum. 63, 1194 (1992).

[20] W. Schmitt, M.Sc. thesis BONN-IR-91-10, Bonn University, 1991.

[21] W.H. Gries, W. Koschig, Surf. Interface Anal. 16, 321 (1990).

[22] W.H. Gries, Microchimica Acta, in press, 1992.

[23] E.W. Becker, W. Ehrfeld, P. Hagmann, A. Maner, D. Münchmeyer, Microelectron. Eng. 4, 35 (1986).

[24] E.W. Becker, W. Ehrfeld, P. Hagmann, A. Maner, D. Münchmeyer, $K f K-$ Bericht 3995, Kernforschungszentrum, Karlsruhe 1985.

[25] KFK Nachrichten, Jahrgang 23, Vol. 2-3/91, Kernforschungszentrum, Karlsruhe 1991, p. 69. 